\title{
STABILITY OF ZN AND CU NANOPARTICLES STUDIED IN AQUEOUS MEDIUM BY SCANNING ELECTRON MICROSCOPY
}

\author{
${ }^{1}$ Pavel SVEC, 'Zuzana BYTESNIKOVA, 1,2Lukas RICHTERA, 1,2Vojtech ADAM \\ ${ }^{1}$ Department of Chemistry and Biochemistry, Mendel University in Brno, Czech Republic, EU \\ ${ }^{2}$ Central European Institute of Technology, Brno University of Technology, Brno, \\ Czech Republic, EU
}

https://doi.org/10.37904/nanocon.2019.8752

\begin{abstract}
The stability of nanoparticles in aquatic environment plays an important role for their environmental and biological application. The storage time has strong impact on the stability of zinc and copper nanoparticles (ZnONPs and CuNPs). The effect of the storage time was studied over time and the results showed a change in morphology of synthesized NPs. The time stability study was divided into three parts: short-term $(0-72 \mathrm{~h})$, medium-term (16 days) and long-term (5 months). In the case of CuNPs the SEM picture revealed the presence of crystal structure. This can be explained as the CuNPs surface layer was oxidized and the crystals on surface are copper oxides. Similar study was conducted on ZnONPs, but in shorter time scale (1 - 3 days). As in the case of CuNPs, ZnONPs showed the same tendency to crystalize. We can conclude that storage time and conditions have a strong impact on the NPs stability.
\end{abstract}

Keywords: Time stability, ZnO nanoparticles, Cu nanoparticles, SEM

\section{INTRODUCTION}

Nanomaterials (NMs) are now widely used in different sectors including agricultural, food, medicinal or military industries and research. Due to their unique properties, using of NMs tend to be more efficient and thus more cost-effective in comparison with traditional approaches [1,2]. The stability of NMs depends on their application and should be monitored in order to achieve their best effect. Therefore, some NMs need to be rapidly degradable, some to maintain their properties as long as possible, while others need to be stable only for a certain period of time. For these reasons, it is necessary to check the quality of the used NMs, not only after synthesis, but also before use, to check whether they changed or decomposed due to storage conditions. Among the wide variety of NMs reported in literature, nanoparticles (NPs) have gained much attention. To monitor the time stability of NPs in the aquatic environment, copper NP (CuNPs) and zinc NPs (ZnONPs) were used. These particles were selected because of their high potential for the agro-industrial applications. CuNPs were synthesized by wet road in our laboratory. The individual testing time intervals were chosen to correlate as best as possible with the actual usage time of specific particles in our laboratories [3].

\section{MATERIALS AND METHODS}

\subsection{Chemicals}

Chemicals used in this study, unless otherwise stated, were purchased from Sigma-Aldrich (St. Louis, MO, USA). The deionized water was prepared using reverse osmosis equipment Aqual 25 (Aqua Osmotic, Tisnov, Czech Republic). The deionized water was further purified by using the apparatus MilliQ Direct QUV, equipped 
with the UV lamp (Aqua Osmotic, Tisnov, Czech Republic). The resistance was $18.2 \mathrm{M} \Omega \cdot \mathrm{cm}$. The pH was measured using pH meter WTW inoLab (Weilheim, Germany).

\subsection{Synthesis of CuNPs}

Synthesis of CuNPs was inspired by Liu et al. [4]. The volume $10 \mathrm{~mL}$ solution of $\mathrm{CuSO}_{4}(0.2 \mathrm{M})$ and a $10 \mathrm{~mL}$ solution of ascorbic acid $(1 \mathrm{M})$ were prepared. The volume of $2.5 \mathrm{~mL}$ sodium carboxymethyl cellulose (4\%; $90 \mathrm{kDa}$ ) was added to the $\mathrm{CuSO}_{4}$ solution. The $\mathrm{pH}$ of both solutions was adjusted to 7 with $5 \% \mathrm{NaOH}$ and $5 \%$ $\mathrm{H}_{2} \mathrm{SO}_{4}$ (Penta, Chrudim, Czech Republic). The $\mathrm{CuSO}_{4}$ solution was stirred at $400 \mathrm{rpm}$ and solution of ascorbic acid was added dropwise. The resulting solution was stirred for 10 minutes. Obtained NPs were washed three times with MilliQ water $(3 \times 150 \mathrm{~mL})$.

\subsection{Synthesis of ZnO NPs}

ZnONPs were received from Mr. Kruszynski. ZnO synthesis was accomplished by coordination compound designed by assembly from hexamethylenetetramine and short-chain carboxylate ions. For $30 \mathrm{~min}$, the mixture was stirred and left to crystalize at room temperature. After 7 - 10 weeks, colourless crystals of precursor were formed [5].

\subsection{Characterization of CuNPs and ZnONPs by SEM}

The morphology and composition were examined by scanning emission microscopy on a Tescan MAIA 3 equipped with a field emission gun (Tescan Ltd., Brno, Czech Republic). Best images were obtained using the In-lens SE detector at working distance between $2.95-3.51 \mathrm{~mm}$ and at $5 \mathrm{kV}$ acceleration voltage. $768 \times 858$ pixel images were obtained at 25,000 - 50,000 fold magnification covering sample area of $4.15-8.31 \mu \mathrm{m}^{2}$. Full frame capture was performed in analytical mode and accumulation of image with image shift correction enabled, and it took about 0.5 minutes with the $\sim 1 \mu \mathrm{s} /$ pixel dwell time. Spot size was set at $2.4 \mathrm{~nm}$.

\section{RESULTS}

Figure 1 shows the structure of ZnONPs from a short-term experiment $(0-72 \mathrm{~h})$. Dry ZnONPs $(\mathrm{A})$ were added to demineralized water and sample was immediately taken for SEM analysis. These freshly dispersed particles (B) show a slight change in the structure in comparison to the original material. ZnONPs begin to recrystallize into larger crystals (green arrows) but also smaller and finer structures are detectable (red arrows). This trend continued with increasing time and there was an increase in the occurrence of larger $\mathrm{ZnO}$ crystals [6,7]. On the other hand, after $24 \mathrm{~h}(\mathrm{C})$ and $72 \mathrm{~h}(\mathrm{D})$, the presence of finer structures decreased significantly (red arrows).

Figure 2 shows the structure of CuNPs from medium-term experiment (16 days). The new CuNPs (A) had a sphere shape and their size was $\sim 1 \mu \mathrm{m}$. Their surface was fine-grained, somewhere smooth. On freshly prepared particles of CuNPs (A) after 16 days began to form small growths of copper oxides, causing greater particle surface roughness [1]. They did not aggregate into larger units or change their spherical shape.

Figure 3 shows the structure of CuNPs from a long-term experiment (5 months). The new CuNPs (A) had a spherical shape and their size was $\sim 300 \mathrm{~nm}$. Their surface was slightly rugged with the appearance of minor surface defects (pits). After 5 months there was a significant change in particle morphology (B). Due to the 
oxidation in the aqueous environment, there was a large increase in the copper oxide crystals on the surface of the particles [1]. Moreover, CuNPs aggregated into larger units and their shape resembled hedgehogs.

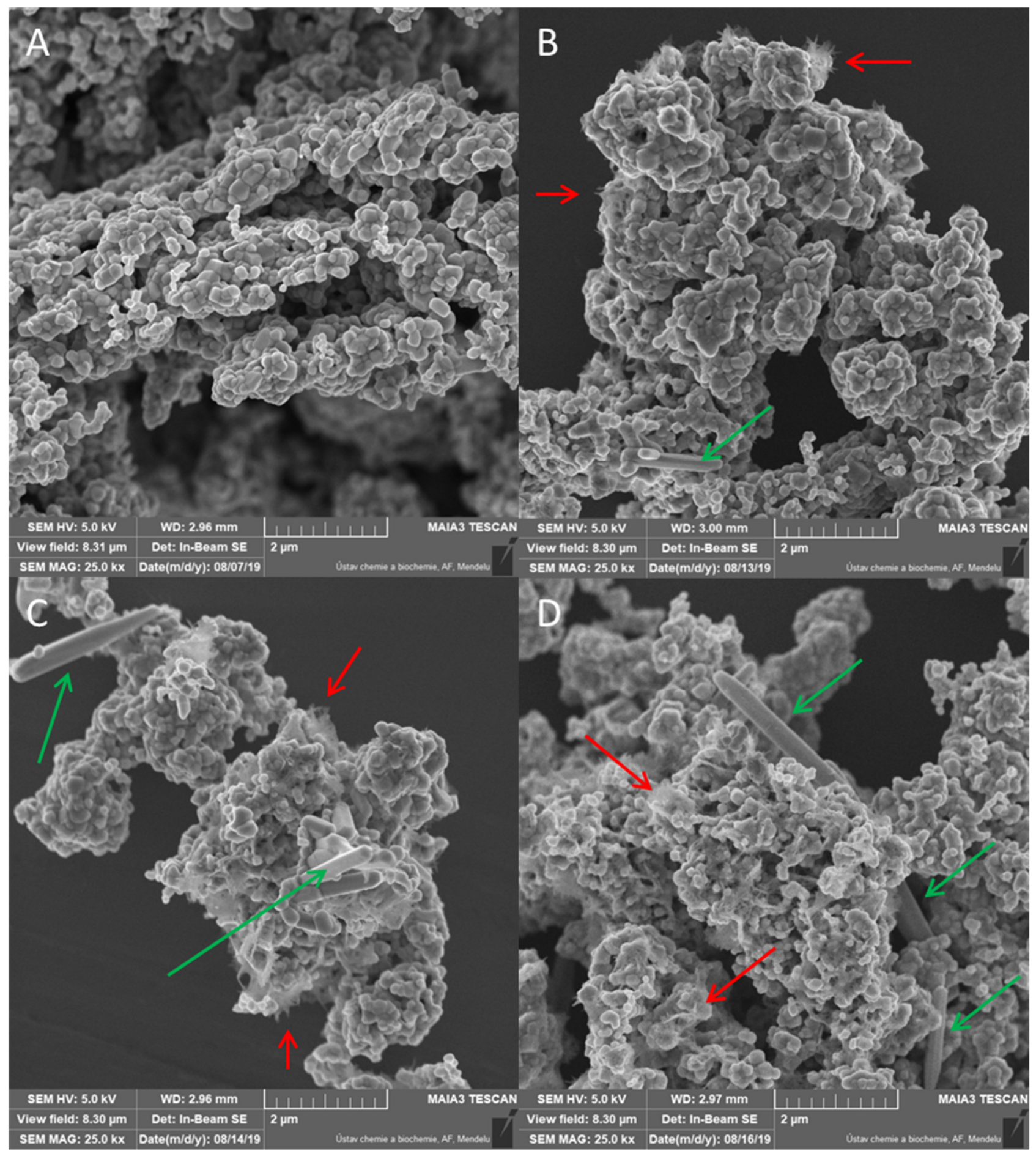

Figure 1 Images of ZnONPs under different time of storage in aquatic environment by using SEM. (A) Dry NPs before put into the MilliQ water. (B) Fresh prepared solution of ZnONPs - time $0 \mathrm{~h}$. (C) ZnONPs after $24 \mathrm{~h}$. (D) ZnONPs after $72 \mathrm{~h}$. 


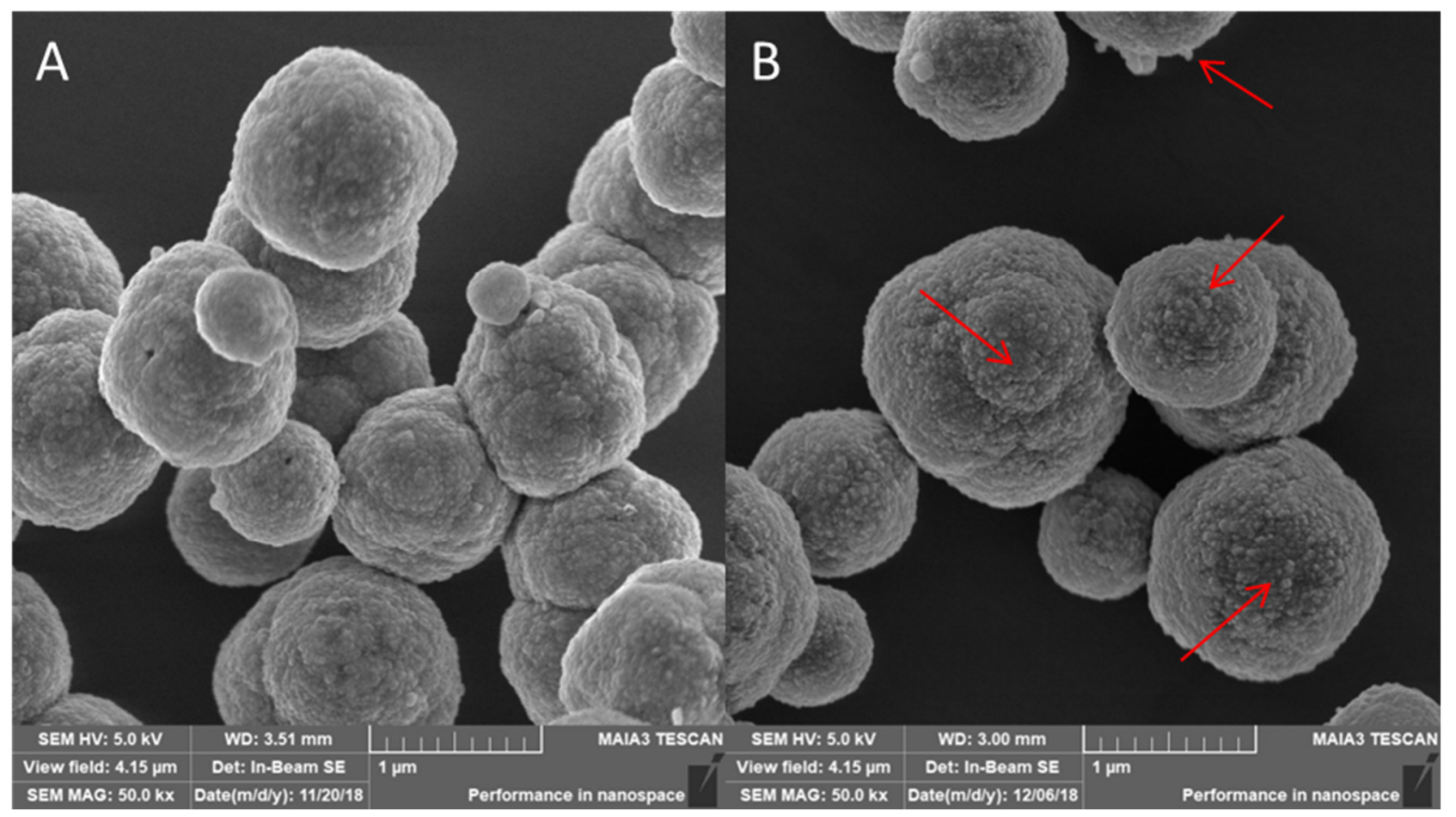

Figure 2 Images of CuNPs under different time of storage in aquatic environment by using SEM. (A) Fresh prepared CuNPs. (B) CuNPs after 16 days.

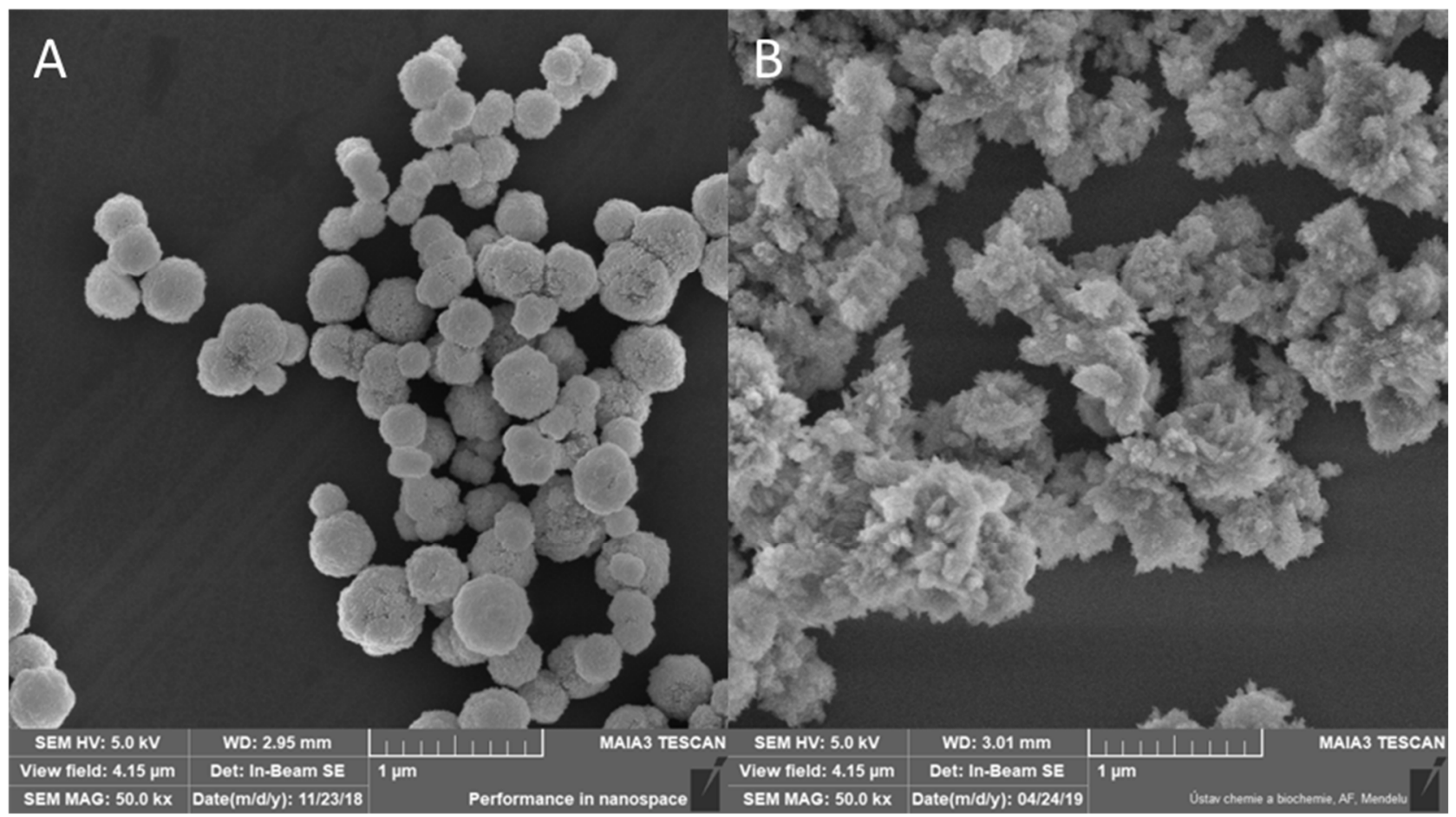

Figure 3 Images of CuNPs under different time of storage in aquatic environment by using SEM.

(A) Fresh prepared CuNPs. (B) CuNPs after 5 months. 


\section{CONCLUSION}

In this work we studied the stability of two kinds of NPs in an aquatic environmental and at different times. As evidenced by the images, the stability of NPs is affected by both time and environment. This was confirmed for ZnONPs, where almost immediately, after the addition of NPs to water, the structure of NPs was changed due to recrystallization. This trend grew with time. More stable and more resistant CuNPs, compared to ZnONPs, began to show slight changes on their surface only after 16 days. However, these changes did not affect the overall morphology of the studied particles or their shape. Smaller CuNPs suffered significant damage in a long-term experiment. The particles aggregated, their surface was strongly oxidized and lost their spherical shape. In conclusion we can say, it is necessary to check the quality of NMs always before using them, because storage or transport condition (ordered commercial NMs) may not be suitable for the material used.

\section{ACKNOWLEDGEMENTS}

This work was supported from ERDF "Multidisciplinary research to increase application potential of nanomaterials in agricultural practice" (No. CZ.02.1.01/0.0/0.0/16_025/0007314).

\section{I would also like to thank Mr. Kruszynski from Institute of General and Ecological Chemistry, Lodz Univerzity of Technology, Poland, for providing ZnONPs.}

\section{REFERENCES}

[1] JARDON-MAXIMINO, N., et al., Oxidation of copper nanoparticles protected with different coatings and stored under ambient conditions. Journal of Nanomaterials, 2018. Art. No. 9512768.

[2] ROMERO-FREIRE, A., et al., Effects of aging and soil properties on zinc oxide nanoparticle availability and its ecotoxicological effects to the earthworm Eisenia Andrei. Environmental Toxikology and Chemistry, 2016. 36(1): p. 137-146.

[3] SEKINE, R., et al., Ageing of dissolved copper and copper-based nanoparticles in five different soils: short term kinetics vs long term fate. Journal of Environmental quality, 2017. 46(6): p. 1198-1205.

[4] LIU, Q.M., et al., Preparation of Cu nanoparticles with ascorbic acid by aqueous solution reduction method. Transactions of Nonferrous Metals Society of China, 2012. 22(9): p. 2198-2203.

[5] KRUSZYNSKI, R. and SWIATKOWSKI, M., The structure of coordination precursors as an effective tool for governing of size and morphology of ZnS and ZnO nanoparticles. Journal of Saudi Chemical Society, 2018. 22(7): p. 816-825.

[6] MAJEDI, M. S., et al., Combined effects of water temperature and chemistry on the environmental fate and behavior of nanosized zinc oxide. Science of the Total Environment, 2014. 496: p. 585-593.

[7] ZHOU, D. X., et al., Increased mobility of metal oxide nanoparticles due tu photo and thermal induced disagglomeration. PLOS ONE, 2012. 7(5): Art. No. e37363. 